\title{
Numerical Computation of Structured Complex Stability Radii of Large-Scale Matrices and Pencils
}

\author{
Peter Benner and Matthias Voigt
}

\begin{abstract}
In this paper we discuss the problem of computing structured complex stability radii of large and sparse matrices and pencils. For this purpose we consider certain structured pseudospectra. To compute the structured complex stability radius we have to find the pseudospectrum which touches the imaginary axis. Therefore, we set up an iteration over the real part of the rightmost pseudoeigenvalue. For that we use a new fast iterative scheme which is based on certain rank1 perturbations of the matrix or pencil. Finally, we illustrate the performance of our algorithm by using real-world example data.
\end{abstract}

\section{INTRODUCTION}

One of the most important properties of dynamical systems is stability. However, even if a system is stable it might be close to instability. This situation might be dangerous, e.g., in the design of feedback controllers. The models we consider are only approximations to real-world phenomena, i.e., they contain modeling errors. The question that arises is whether the feedback controller we have designed based on the model indeed stabilizes the dynamics of the real-world problem. In other words, the question is how robust is the controller with respect to perturbations; or how much can we perturb the controller such that the closed-loop system is still stable?

We consider linear time-invariant systems of the form

$$
\dot{x}(t)=A x(t)+B u(t), \quad y(t)=C x(t),
$$

where $A \in \mathbb{R}^{n \times n}, B \in \mathbb{R}^{n \times m}, C \in \mathbb{R}^{p \times n}$, and $x(t) \in \mathbb{R}^{n}$ is the state vector, $u(t) \in \mathbb{R}^{m}$ is the control input vector, and $y(t) \in \mathbb{R}^{p}$ is the output vector. We furthermore assume that all involved matrices are large and sparse and that $m, p \ll n$. Such systems are called (asymptotically) stable if and only if the matrix $A$ is stable, i.e., $\Lambda(A) \subset \mathbb{C}^{-}:=$ $\{z \in \mathbb{C}: \operatorname{Re} z<0\}$, where $\Lambda(A)$ denotes the spectrum of $A$. To measure the robustness of the system against perturbations, i.e., the "distance to instability", unstructured and structured stability radii have been introduced in [1]-[3]. In this paper we focus on structured stability radii which measure the robustness of a closed-loop system

$$
\dot{x}(t)=A_{K} x(t):=(A+B K C) x(t),
$$

with respect to perturbations in the controller $K$. These perturbations lead to the closed-loop system

$$
\begin{aligned}
\dot{x}(t)=A_{K+\Delta} x(t): & =(A+B(K+\Delta) C) x(t) \\
& =\left(A_{K}+B \Delta C\right) x(t),
\end{aligned}
$$

P. Benner and M. Voigt are with the Max Planck Institute for Dynamics of Complex Technical Systems, Sandtorstr. 1, 39106 Magdeburg, Germany. Emails: benner@mpi-magdeburg.mpg.de, voigtm@mpi-magdeburg.mpg.de. where $\Delta \in \mathbb{C}^{m \times p}$ is the perturbation matrix. Therefore, the structured complex stability radius of the stable matrix $A$ (we replace $A_{K}$ by $A$ for simplicity) with respect to $B$ and $C$ is defined by

$$
\begin{array}{r}
r_{\mathbb{C}}(A, B, C):=\inf \left\{\|\Delta\|_{2}: \Lambda(A+B \Delta C) \cap \mathrm{i} \mathbb{R} \neq \emptyset\right. \\
\text { with } \left.\Delta \in \mathbb{C}^{m \times p}\right\} .
\end{array}
$$

Note, that structured stability radii can be defined for arbitrary norms of the perturbation $\Delta$. In this paper we stick to the 2-norm $\|\cdot\|_{2}$ as it is of largest practical relevance.

We also consider generalizations of $r_{\mathbb{C}}(A, B, C)$ for $m a$ trix pencils $\lambda E-A$. These pencils typically arise in the analysis of dynamical systems of the form

$$
E \dot{x}(t)=A x(t)+B u(t), \quad y(t)=C x(t),
$$

where $E \in \mathbb{R}^{n \times n}$ is allowed to be a singular matrix. However, we assume that $\lambda E-A$ is regular, i.e., $\operatorname{det}(\lambda E-$ $A) \not \equiv 0$. In this context we speak about differential-algebraic equations or descriptor systems [4], [5]. Assume that $\lambda E-A$ is stable, i.e., $\Lambda_{\mathrm{f}}(E, A) \subset \mathbb{C}^{-}$, where $\Lambda_{\mathrm{f}}(E, A)$ denotes the finite spectrum of $\lambda E-A$. The definition of the structured complex stability radius $r_{\mathbb{C}}(E, A, B, C)$ for such matrix pencils is rather involved and will therefore be discussed in the next section.

We briefly discuss associated concepts in the frequency domain. Consider the transfer function of (1), defined by

$$
G(z)=C(z E-A)^{-1} B .
$$

We call $G(z)$ proper if $\lim _{\omega \rightarrow \infty}\|G(\mathrm{i} \omega)\|_{2}<\infty$, otherwise we call it improper. By $\mathcal{R} \mathcal{H}_{\infty}^{p \times m}$ we denote the rational Banach space of all stable and proper transfer functions of the form (2), see [6]. For this space we define the $\mathcal{H}_{\infty}$-norm, given by

$$
\|G\|_{\mathcal{H}_{\infty}}:=\sup _{z \in \mathbb{C}^{+}} \sigma_{\max }(G(z))=\sup _{\omega \in \mathbb{R}} \sigma_{\max }(G(\mathrm{i} \omega)),
$$

with $\mathbb{C}^{+}:=\{z \in \mathbb{C}: \operatorname{Re} z>0\}$. In [7] we show that the relation

$$
\|G\|_{\mathcal{H}_{\infty}}= \begin{cases}1 / r_{\mathbb{C}}(E, A, B, C), & \text { if } G \not \equiv 0 \\ \infty, & \text { if } G \equiv 0\end{cases}
$$

holds.

Numerical methods for computing structured stability radii (and equivalently $\mathcal{H}_{\infty}$-norms) have been known for a long time. Most of them are based on relations between stability radii and the spectrum of certain Hamiltonian matrices or pencils. For an overview, we refer to [8]-[13]. 
The most general algorithm [12] iterates over certain skewHamiltonian/Hamiltonian matrix pencils and determines the finite, purely imaginary eigenvalues in each step. The drawback of the algorithm is the decision whether there are such eigenvalues. It is important to find all of them since otherwise the algorithm could fail. In [12] this issue was addressed by using a structure-preserving eigensolver (real-case version of [14]), which prevents the finite, purely imaginary eigenvalues to move off the imaginary axis as long as their pairwise distance is sufficiently large. However, this method computes a full structured factorization of the pencil in each step. Due to its cubic complexity it is infeasible for large-scale problems. We could consider a closely related extended even matrix pencil and try to compute the desired eigenvalues close to a specified shift with the method presented in [15]. However, the question remains how to reliably compute all finite, purely imaginary eigenvalues. Therefore, in this paper we use another approach based on pseudospectra. Recently, also a similar method based on optimization over so-called spectral value sets was developed [16]. However, it does not take descriptor systems into account and uses a different optimization technique.

\section{Structured Complex Stability Radil For MATRIX PENCILS}

We turn now to the definition of the structured complex stability radius for matrix pencils which requires the introduction of several mathematical and control theoretic terms.

A regular matrix pencil $\lambda E-A$ can be reduced to Weierstraß canonical form [5]

$$
E=W\left[\begin{array}{cc}
I_{n_{\mathrm{f}}} & 0 \\
0 & N
\end{array}\right] T, \quad A=W\left[\begin{array}{cc}
J & 0 \\
0 & I_{n_{\infty}}
\end{array}\right] T,
$$

where $W$ and $T$ are nonsingular, $I_{k}$ is an identity matrix of order $k, J$ and $N$ are in Jordan canonical form and $N$ is nilpotent with index of nilpotency $\nu$. The number $\nu$ is also called the algebraic index of the descriptor system (1) and $n_{\mathrm{f}}$ and $n_{\infty}$ are the dimensions of the deflating subspaces of $\lambda E-A$ corresponding to the finite and infinite eigenvalues, respectively. We say that a finite or infinite eigenvalue is defective, if one of the corresponding Jordan blocks in $J$ or $N$ is larger than one. By using the transformation matrices $W$ and $T$, we can also write $B$ and $C$ as

$$
B=W^{-1}\left[\begin{array}{l}
B_{1} \\
B_{2}
\end{array}\right], \quad C=\left[\begin{array}{ll}
C_{1} & C_{2}
\end{array}\right] T^{-1},
$$

to obtain an equivalent descriptor system.

Furthermore we need some controllability and observability concepts [4]. The descriptor system (1) is called

- completely controllable, if $\operatorname{rank}\left[\begin{array}{ll}\lambda E-A & B\end{array}\right]=n$ for all $\lambda \in \mathbb{C}$ and furthermore $\operatorname{rank}\left[\begin{array}{ll}E & B\end{array}\right]=n$;

- completely observable, if $\operatorname{rank}\left[\begin{array}{c}\lambda E-A \\ C\end{array}\right]=n$ for all $\lambda \in \mathbb{C}$ and furthermore rank $\left[\begin{array}{l}E \\ C\end{array}\right]=n$.

We can also define these concepts for single eigenvalues of $\lambda E-A$. A descriptor system (1) is called
- controllable at $\lambda \in \mathbb{C}$ if $\operatorname{rank}\left[\begin{array}{ll}\lambda E-A & B\end{array}\right]=n$;

- controllable at $\lambda=\infty$ if $\operatorname{rank}\left[\begin{array}{ll}E & B\end{array}\right]=n$;

- observable at $\lambda \in \mathbb{C}$ if $\operatorname{rank}\left[\begin{array}{c}\lambda E-A \\ C\end{array}\right]=n$;

- observable at $\lambda=\infty$ if $\operatorname{rank}\left[\begin{array}{l}E \\ C\end{array}\right]=n$;

otherwise it is called uncontrollable or unobservable at $\lambda$, respectively. Note that in the above definition, one can also consider each individual Jordan block of the Weierstraß canonical form separately in case of multiple eigenvalues. This is possible by considering the corresponding eigenvectors. Let $x$ and $y$ be right and left eigenvectors corresponding to a Jordan block in $J$ or $N$ of (4). Then

$y^{H}\left[\begin{array}{ll}\lambda E-A & B\end{array}\right]=\left[\begin{array}{ll}0 & y^{H} B\end{array}\right], \quad\left[\begin{array}{c}\lambda E-A \\ C\end{array}\right] x=\left[\begin{array}{c}0 \\ C x\end{array}\right]$.

We say that such a Jordan block is controllable if $B^{T} y \neq 0$, and observable if $C x \neq 0$, otherwise we call it uncontrollable or unobservable. We use this block-wise definition when talking about controllability and observability of eigenvalues.

Now we define the numbers

$$
\begin{aligned}
& r_{\mathbb{C}}^{\mathrm{f}}(E, A, B, C):=\inf \left\{\|\Delta\|_{2}:\right. \\
&\left.\Lambda_{\mathrm{f}}(E, A+B \Delta C) \cap \mathrm{i} \mathbb{R} \neq \emptyset \text { with } \Delta \in \mathbb{C}^{m \times p}\right\}, \\
& r_{\mathbb{C}}^{\infty}(E, A, B, C):=\inf \left\{\|\Delta\|_{2}:\right. \\
& \Lambda_{\infty}(E, A+B \Delta C) \text { with } \Delta \in \mathbb{C}^{m \times p} \text { has }
\end{aligned}
$$

controllable and observable defective eigenvalues

$$
\text { or } \lambda E-(A+B \Delta C) \text { is a singular pencil }\} \text {, }
$$

where $\Lambda_{\infty}(E, A)$ is the set of infinite eigenvalues of $\lambda E-$ $A$ (counting multiplicity). Then we define the structured complex stability radius by

$$
\begin{aligned}
r_{\mathbb{C}}(E, A, B, C):=\min \left\{r_{\mathbb{C}}^{\mathrm{f}}(E, A, B, C),\right. & \\
& \left.r_{\mathbb{C}}^{\infty}(E, A, B, C)\right\} .
\end{aligned}
$$

The value of $r_{\mathbb{C}}^{\mathrm{f}}(E, A, B, C)$ is the size of the smallest structured perturbation that makes the system unstable. The interpretation of $r_{\mathbb{C}}^{\infty}(E, A, B, C)$ is more involved. Defective infinite eigenvalues do not directly make the system unstable. However, if there are controllable and observable ones we can construct an arbitrarily small structured perturbation such that the system will be unstable. This means that systems with controllable and observable defective infinite eigenvalues are on the "boundary to instability". If the perturbed matrix pencil becomes singular, then all complex numbers are eigenvalues and the system dynamics is not well-defined.

It is easy to see that $r_{\mathbb{C}}(A, B, C)=r_{\mathbb{C}}\left(I_{n}, A, B, C\right)$. Therefore, we stick to the more general concept in this paper.

\section{Derivation of the Algorithm}

In this section we derive the theoretical basis of our algorithm. Therefore, we compute both $r_{\mathbb{C}}^{\infty}(E, A, B, C)$ and $r_{\mathbb{C}}^{\mathrm{f}}(E, A, B, C)$ independent of each other. The computation of $r_{\mathbb{C}}^{\infty}(E, A, B, C)$ can be done by employing the following lemma [7]: 
Lemma 1. It holds that

$$
r_{\mathbb{C}}^{\infty}(E, A, B, C)= \begin{cases}1 / \lim _{\omega \rightarrow \infty} \sigma_{\max }(G(\mathrm{i} \omega)) & \text { if } G \not \equiv 0, \\ \infty & \text { if } G \equiv 0 .\end{cases}
$$

For the computation of $r_{\mathbb{C}}^{\mathrm{f}}(E, A, B, C)$ we need some further mathematical terms. The structured $\varepsilon$-pseudospectrum of the pencil $\lambda E-A$ with respect to $B$ and $C$ is defined by

$$
\begin{array}{r}
\Lambda_{\varepsilon}(E, A, B, C)=\left\{z \in \mathbb{C}: z \in \Lambda_{\mathrm{f}}(E, A+B \Delta C)\right. \\
\text { for some } \left.\Delta \in \mathbb{C}^{m \times p} \text { with }\|\Delta\|_{2}<\varepsilon\right\} .
\end{array}
$$

Furthermore, we need the structured $\varepsilon$-pseudospectral abscissa, given by

$$
\alpha_{\varepsilon}(E, A, B, C):=\max \left\{\operatorname{Re} z: z \in \Lambda_{\varepsilon}(E, A, B, C)\right\} .
$$

From these definitions it is clear that $\alpha_{r_{\mathrm{C}}^{\mathrm{f}}}(E, A, B, C)=0$. So the main idea of our algorithm is to find the (unique) root of the function $\alpha(\varepsilon):=\alpha_{\varepsilon}(E, A, B, C)$. To get an efficient algorithm we need to evaluate $\alpha(\varepsilon)$ for different values of $\varepsilon$ in a cheap way.

\section{A. Computation of the Structured $\varepsilon$-Pseudospectral Abscissa}

In this subsection we derive a fast algorithm for computing $\alpha(\varepsilon)$. We assume that the pseudospectra of interest are not the whole complex plane, i.e., $\varepsilon$ is smaller than the smallest norm of a structured perturbation that makes the pencil singular. The following fundamental result is a generalization of the corresponding one in [1] and is proven in [7].

Theorem 1. Let $z \in \mathbb{C} \backslash \Lambda_{\mathrm{f}}(E, A)$ be given and $\varepsilon>0$. Then the following statements are equivalent:

(a) $z \in \Lambda_{\varepsilon}(E, A, B, C)$.

(b) $\sigma_{\max }(G(z))>\varepsilon^{-1}$.

(c) There exist vectors $u \in \mathbb{C}^{m}$ and $v \in \mathbb{C}^{p}$ with $\|u\|_{2}<1$ and $\|v\|_{2}<1$ such that $z \in \Lambda_{\mathrm{f}}\left(E, A+\varepsilon B u v^{H} C\right)$.

From Theorem 1 we can conclude that

$$
\Lambda_{\varepsilon}(E, A, B, C)=\left\{z \in \mathbb{C}: \sigma_{\max }(G(z))>\varepsilon^{-1}\right\},
$$

including the poles of $G(z)$. The boundary is given by

$$
\partial \Lambda_{\varepsilon}(E, A, B, C)=\left\{z \in \mathbb{C}: \sigma_{\max }(G(z))=\varepsilon^{-1}\right\} .
$$

In other words, also the rightmost pseudoeigenvalue is arbitrarily close to the curve $\partial \Lambda_{\varepsilon}(E, A, B, C)$. Thus, our strategy consists of computing a sequence of suitable structured rank1 perturbed pencils $\lambda E-\left(A+\varepsilon B u v^{H} C\right)$ such that one of the perturbed eigenvalues converges to the rightmost pseudoeigenvalue of $\lambda E-A$. A similar technique has already been successfully applied to compute the pseudospectral abscissa of a matrix, see [17]. We need the following result for the first order perturbation theory of matrix pencils.

Theorem 2. [18] Let $\lambda E-A \in \mathbb{C}^{n \times n}$ be a given matrix pencil and let $x, y \in \mathbb{C}^{n}$ be right and left eigenvectors corresponding to a simple finite eigenvalue $\lambda=\frac{y^{H} A x}{y^{H} E x}$. Let $\lambda E-\left(A+t B u v^{H} C\right)$ be a perturbed matrix pencil with eigenvalue $\tilde{\lambda}$. Then it holds

$$
\tilde{\lambda}=\lambda+t \frac{y^{H} B u v^{H} C x}{y^{H} E x}+\mathcal{O}\left(t^{2}\right) .
$$

Furthermore, Theorem 2 directly yields

$$
\left.\frac{\mathrm{d} \tilde{\lambda}(t)}{\mathrm{d} t}\right|_{t=0}=\frac{y^{H} B u v^{H} C x}{y^{H} E x} .
$$

Now, we describe how such rank-1 perturbations can be constructed in an optimal way. Therefor, let $\lambda E-A$ with a simple finite eigenvalue $\lambda$ and corresponding right and left eigenvectors $x, y$ with $y^{H} E x>0$ be given. Furthermore let $u \in \mathbb{C}^{m}$ and $v \in \mathbb{C}^{p}$ with $\|u\|_{2}=\|v\|_{2}=1$ be given vectors. Then, it holds

$$
\begin{aligned}
\operatorname{Re}\left(\left.\frac{\mathrm{d} \tilde{\lambda}(t)}{\mathrm{d} t}\right|_{t=0}\right) & =\frac{\operatorname{Re}\left(y^{H} B u v^{H} C x\right)}{y^{H} E x} \\
& \leq \frac{\left\|y^{H} B\right\|_{2}\|C x\|_{2}}{y^{H} E x} .
\end{aligned}
$$

Equality in (6) holds for $u=\frac{B^{T} y}{\left\|B^{T} y\right\|_{2}}, v=\frac{C x}{\|C x\|_{2}}$. Hence, local maximal growth in $\operatorname{Re}(\tilde{\lambda}(t))$ as $t$ increases from 0 is achieved for this choice of $u$ and $v$. In this way we generate the initial perturbation. Next, we consider subsequent perturbations. For this, let $\lambda E-\hat{A}:=\lambda E-\left(A+\varepsilon B \hat{u} \hat{v}^{H} C\right)$ with a simple finite eigenvalue $\hat{\lambda}$ and associated right and left eigenvectors $\hat{x}, \hat{y}$ with $\hat{y}^{H} E \hat{x}>0$ be the perturbed matrix pencil. Let in addition the vectors $u \in \mathbb{C}^{m}, v \in \mathbb{C}^{p}$ with $\|u\|_{2}=\|v\|_{2}=1$ be given. We consider the family of perturbations of the matrix pencil $\lambda E-\hat{A}$ of the form

$$
\lambda E-\left(\hat{A}+t B\left(u v^{H}-\hat{u} \hat{v}^{H}\right) C\right),
$$

which are structured $\varepsilon$-norm rank-1 perturbations of $\lambda E-$ $A$ for $t=0$ and $t=\varepsilon$. For the perturbed eigenvalue, for simplicity called again $\tilde{\lambda}$, we obtain

$$
\begin{aligned}
\operatorname{Re}\left(\left.\frac{\mathrm{d} \tilde{\lambda}(t)}{\mathrm{d} t}\right|_{t=0}\right) & =\frac{\operatorname{Re}\left(\hat{y}^{H} B\left(u v^{H}-\hat{u} \hat{v}^{H}\right) C \hat{x}\right)}{\hat{y}^{H} E \hat{x}} \\
& \leq \frac{\left\|\hat{y}^{H} B\right\|_{2}\|C \hat{x}\|_{2}-\operatorname{Re}\left(\hat{y}^{H} B \hat{u} \hat{v}^{H} C \hat{x}\right)}{\hat{y}^{H} E \hat{x}} .
\end{aligned}
$$

Similarly as above, equality in (7) holds for $u=\frac{B^{T} \hat{y}}{\left\|B^{T} \hat{y}\right\|_{2}}$, $v=\frac{C \hat{x}}{\|C \hat{x}\|_{2}}$. Then the right-hand-side of (7) is larger or equal than zero. Thus, a nonnegative local maximal growth in the real part of $\tilde{\lambda}(t)$ is achieved as $t$ increases from 0 . So, the basic algorithm consists of successively choosing an eigenvalue and constructing the perturbations described above by using the corresponding eigenvectors. However, an important question is how to actually choose these eigenvalues. This will be discussed in the next subsection. 


\section{B. Choice of the Eigenvalues}

Recall that we want to construct structured $\varepsilon$-norm rank1 perturbations of the pencil $\lambda E-A$ such that one of the perturbed eigenvalues converges to the rightmost $\varepsilon$ pseudoeigenvalue. Intuitively, in each step one would choose the rightmost eigenvalue of the perturbed pencil to construct the next perturbation. However, that might not be a good choice. Note that the perturbability of an eigenvalue $\lambda$ with right and left eigenvectors $x$ and $y$ highly depends on $\left\|B^{T} y\right\|_{2}$ and $\|C x\|_{2}$. If these values are small, no large perturbation is possible. We remark that these values are also related to the classical definitions of controllability and observability of $\lambda$ in a dynamical system of the form (1). Roughly speaking, the "larger" $\left\|B^{T} y\right\|_{2}$ is, the "larger" is the distance of the system to uncontrollability at $\lambda$. So, large values of $\left\|B^{T} y\right\|_{2}$ indicate a good controllability at $\lambda$. Similar considerations can also be made for observability.

Consequently, for our algorithm we look for eigenvalues that have both sufficiently large real part and a high controllability and observability. An algorithm which unites both concepts and can compute the desired eigenvalues is the (subspace accelerated MIMO) dominant pole algorithm (SAMDP), introduced by Rommes and Martins [19]-[22]. The algorithm is actually designed to find the poles which have the highest influence on the frequency response of the transfer function $G(z)$. Assume that $\lambda E-A$ has only simple finite eigenvalues $\lambda_{k}$ with left and right eigenvectors $y_{k}$ and $x_{k}$, normalized such that $y_{k}^{H} E x_{k}=1$. Then it holds

$$
G(z)=\sum_{k=1}^{n} \frac{R_{k}}{z-\lambda_{k}}+R_{\infty}
$$

with the residues

$$
R_{k}=C x_{k} y_{k}^{H} B, \text { and } R_{\infty}=\lim _{\omega \rightarrow \infty} G(\mathrm{i} \omega) .
$$

Thus, $\left\|R_{k}\right\|_{2}=\left\|C x_{k}\right\|_{2}\left\|B^{T} y_{k}\right\|_{2}$ is a measure for simultaneous controllability and observability of $\lambda_{k}$. We observe that if $\lambda_{j}$ is close to the imaginary axis and $\left\|R_{j}\right\|_{2}$ is large, then for $\omega \approx \operatorname{Im}\left(\lambda_{j}\right)$ it holds

$$
G(\mathrm{i} \omega) \approx \frac{R_{j}}{-\operatorname{Re}\left(\lambda_{j}\right)}+\sum_{\substack{k=1 \\ k \neq j}}^{n} \frac{R_{k}}{\mathrm{i} \omega-\lambda_{k}}+R_{\infty},
$$

and therefore $\|G(\mathrm{i} \omega)\|_{2}$ is large, too. These considerations give the motivation for the following definition. We call an eigenvalue $\lambda_{j} \in \Lambda_{\mathrm{f}}(E, A)$ dominant pole of $G(z)$, if

$$
\frac{\left\|R_{k}\right\|_{2}}{\left|\operatorname{Re}\left(\lambda_{k}\right)\right|}<\frac{\left\|R_{j}\right\|_{2}}{\left|\operatorname{Re}\left(\lambda_{j}\right)\right|}, \quad k=1, \ldots, n, \quad k \neq j .
$$

The most dominant poles can be determined by SAMDP and are essentially what we are looking for. However, we also deal with positive structured pseudospectral abscissae. By using the definition (8), the eigenvalues tend to loose dominance as soon as they have crossed the imaginary axis into the right half-plane. Then, in subsequent iterations, eigenvalues in the left half-plane tend to be determined as most dominant. This is of course an undesired behavior since this could lead to convergence problems when the rightmost pseudoeigenvalue is "far" in the right half-plane. Therefore, we use an alternative dominance measure which does not have this drawback. We call an eigenvalue $\lambda_{j} \in \Lambda_{\mathrm{f}}(E, A)$ exponentially dominant pole of $G(z)$, if

$$
\begin{array}{r}
\left\|R_{k}\right\|_{2} \exp \left(\beta \operatorname{Re}\left(\lambda_{k}\right)\right)<\left\|R_{j}\right\|_{2} \exp \left(\beta \operatorname{Re}\left(\lambda_{j}\right)\right), \\
k=1, \ldots, n, \quad k \neq j .
\end{array}
$$

The parameter $\beta$ is a weighting factor which defines the trade-off between the influence of the residue and real part of the eigenvalues. In our numerical experiments it turned out that $\beta=100$ is a good choice for many examples (high weight on the real part). Since SAMDP delivers the poles which have the highest influence on the frequency response of a system and due to the relation (3), we can determine very good initial estimates for $r_{\mathbb{C}}^{\mathrm{f}}(E, A, B, C)$. We compute some of the dominant poles $\lambda_{k}, k=1, \ldots, \ell$, and determine an estimate $r_{\mathbb{C}}^{\text {est }}(E, A, B, C)$ as

$$
r_{\mathbb{C}}^{\text {est }}(E, A, B, C)=1 / \max _{1 \leq k \leq \ell} \sigma_{\max }\left(G\left(\mathrm{i} \omega_{k}\right)\right)
$$

with $\omega_{k}=\operatorname{Im}\left(\lambda_{k}\right), k=1, \ldots, \ell$.

\section{Algorithmic Details}

In this subsection we present some pseudocode of the algorithms that have been derived. Algorithm 1 summarizes the procedure for the computation of the structured $\varepsilon$-pseudospectral abscissa. In our implementation of the algorithm, we use estimates of the eigenvectors $x$ and $y$ that are used to construct the optimal perturbation. This is possible since we can take the eigenvectors returned by the previous $\alpha(\varepsilon)$-evaluations of the root-finding algorithm. This accelerates the computation drastically since in the final rootfinding steps the eigenvectors also converge to $x$ and $y$.

We mention the drawback that the algorithm not necessarily converges to the globally rightmost value on the boundary of the $\varepsilon$-pseudospectrum $\partial \Lambda_{\varepsilon}$ in (5). Mostly it does but in some rare situations the algorithm converges only to a local maximizer. This especially happens in the first iteration of the root-finding algorithm when no good estimates of the optimal eigenvectors are available. Therefore, sometimes one has to try several dominant poles to find the global maximizer in the beginning. To find the root of $\alpha(\varepsilon)$, we use a simple secant method which has a superlinear convergence with convergence rate $\frac{1+\sqrt{5}}{2} \approx 1.618$. A comparison of different standard root-finding methods and standard software to compute structured complex stability radii is presented in [23]. As the numerical examples show, we only need very few (3-5) iterations of this method in most cases to find the root with a sufficient accuracy. When computing the dominant poles of the system we already obtain a very good estimate of $r_{\mathbb{C}}^{\text {est }}(E, A, B, C)$ as in (10) which is used as an initial value for the root-finder.

\section{NUMERICAL RESUlTS}

In this section be present some numerical results of our implementations. The tests have been performed on a 2.6.32-23generic-pae Ubuntu machine with Intel ${ }^{\circledR} \mathrm{Core}^{\mathrm{TM}} 2$ Duo CPU 


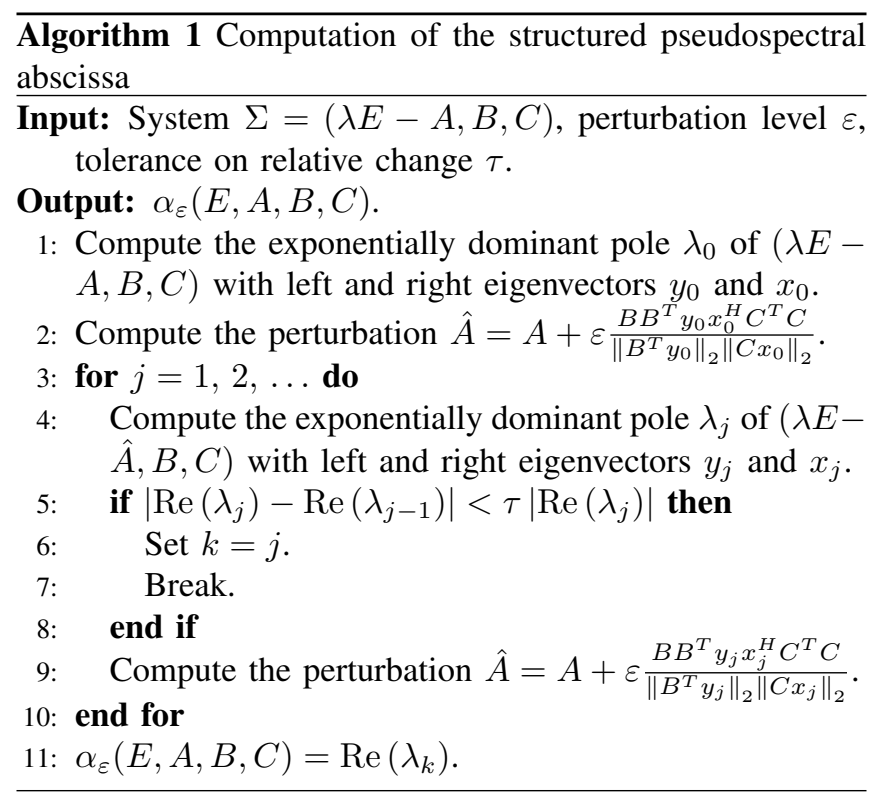

with $3.00 \mathrm{GHz}$ and $2 \mathrm{~GB}$ RAM. The algorithms have been implemented and tested in MATLAB 7.11.0.584 (R2010b). To compute the (exponentially) dominant poles we use a (modified) MATLAB implementation by Rommes ${ }^{1}$. The data for the numerical examples was taken from [21], [24]-[26], and most of it can also be downloaded from Rommes' website. All systems, except the PISection examples (\# 2-17 in Table I) have stable matrix pencils $\lambda E-A$. The PISection pencils have zero eigenvalues, therefore we slightly shift the spectrum into the left half-plane by using the modified pencils $\lambda E-\left(A-10^{-8} E\right)$. For the dominance measure in (9) we use $\beta=100$. The tolerance on the relative change of the iterates in Algorithm 1 is set to $\tau=10^{-3}$. We also abort the iteration when the iterates are smaller than $1000 \mathrm{u}$, where $\mathbf{u}$ denotes the machine precision, or when the iterates start to cycle, which typically happens when they are approaching zero. In the root finder we abort this iteration when the relative change between two subsequent iterates is below $10^{-6}$. To obtain $r_{\mathbb{C}}^{\text {est }}(E, A, B, C)$, we compute 20 dominant poles using the original version of SAMDP. For every further outer iteration we compute only 5 dominant poles using the modified algorithm with the exponential dominance measure.

In Table I we summarize the results of 28 numerical tests. The first 9 examples are standard or generalized state space systems whereas the other 19 ones are descriptor systems. By $n_{\text {outer }}$ we denote the number of outer iterations, i.e., the number of $\alpha(\varepsilon)$-evaluations to find the root. By $n_{\text {inner }}$ we refer to the total number of inner iterations, i.e., the total number of steps needed by Algorithm 1.

For all tests, the correct value of $r_{\mathbb{C}}(E, A, B, C)$ is found. In 25 tests, the first outer iteration returns a positive value. However, for 3 tests (M10PI_n1, M10PI_n, bips07_1693), a negative value is returned and therefore we have to try more dominant poles (one for each M10PI_n1, M10PI_n and three for bips07_1693) to

\footnotetext{
${ }^{1}$ http://sites.google.com/site/rommes/software
}

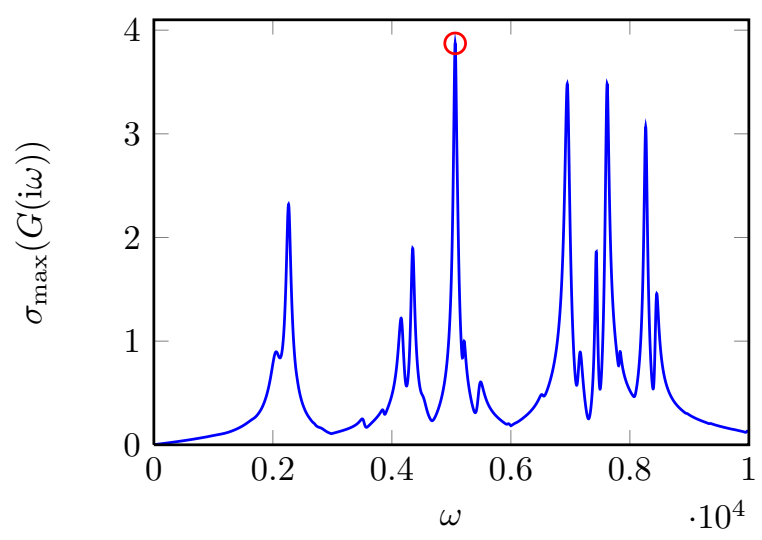

Fig. 1. Transfer function plot for the M20PI_n test example with computed $\mathcal{H}_{\infty}$-norm (red circle)

converge to the correct initial value. Note also that the convergence speed of the algorithm highly depends on the properties of the examples, e.g., for bips07_1998, the convergence is significantly slower than for the rest in the bips 07 group, although they are comparable in size. Finally, in Figure 1 the transfer function for the M20PI_n example is evaluated on the imaginary axis including the computed $\mathcal{H}_{\infty}$-norm (see (3)). Our algorithm computes the correct value, even though there are lots of close-by peaks of similar height. Unfortunately, there are also examples that our algorithm cannot solve. These include systems whose transfer functions only have real poles or those where the structured pseudospectra have lots of very close locally rightmost points on the boundary, see [7] for more details.

\section{Conclusions and Future Research PERSPECTIVES}

In this paper we have introduced a new iterative scheme for computing the structured complex stability radius of a matrix or a pencil, based on the method introduced in [17]. The algorithm computes a sequence of structured pseudospectral abscissae. This is done by computing an optimal rank-1 perturbation of the system such that one of the eigenvalues of the perturbed matrix or pencil converges to the rightmost pseudoeigenvalue. This algorithm can be seen as a basis to solve certain related problems. Open questions concern the computation of unstructured stability radii. Another interesting problem is the computation of real stability radii where the perturbation matrix is assumed to be real. Finally, we mention the passivity radius of a dynamical system which might also be put into this framework.

\section{ACKNOWLEDGEMENT}

We would like to thank Volker Mehrmann for pointing out possible issues with perturbations of infinite eigenvalues.

\section{REFERENCES}

[1] D. Hinrichsen and A. J. Pritchard, "Stability radii of linear systems," Syst. Control Lett., vol. 7, pp. 1-10, July 1986.

[2] _ "Stability radius for structured perturbations and the algebraic Riccati equation," Syst. Control Lett., vol. 8, pp. 105-113, Aug. 1986. 
TABLE I

NUMERICAL RESULTS FOR 28 TEST EXAMPLES

\begin{tabular}{|c|c|c|c|c|c|c|c|c|c|}
\hline$\#$ & example & $n$ & $m$ & $p$ & $r_{\mathbb{C}}(E, A, B, C)$ & $\alpha_{r_{\mathbb{C}}}(E, A, B, C)$ & $n_{\text {outer }}$ & $n_{\text {inner }}$ & time in $\mathrm{s}$ \\
\hline 1 & CDplayer & 120 & 2 & 2 & $4.31068 \mathrm{e}-07$ & $-3.0943 \mathrm{e}-15$ & 3 & 9 & 2.92 \\
\hline 2 & S10PI_n1 & 528 & 1 & 1 & $2.51602 \mathrm{e}-01$ & $1.8451 \mathrm{e}-12$ & 4 & 14 & 3.22 \\
\hline 3 & S20PI_n1 & 1028 & 1 & 1 & $2.90430 \mathrm{e}-01$ & $6.3894 \mathrm{e}-12$ & 4 & 15 & 5.16 \\
\hline 4 & S40PI_n1 & 2028 & 1 & 1 & $2.98747 \mathrm{e}-01$ & $1.3026 \mathrm{e}-10$ & 4 & 18 & 9.43 \\
\hline 5 & S 80PI_n1 & 4028 & 1 & 1 & $2.96722 \mathrm{e}-01$ & $4.1804 \mathrm{e}-13$ & 4 & 14 & 15.74 \\
\hline 6 & M10PI_n1 & 528 & 3 & 3 & $2.46511 \mathrm{e}-01$ & $3.9342 \mathrm{e}-13$ & 5 & 18 & 4.64 \\
\hline 7 & M20P I_n1 & 1028 & 3 & 3 & $2.58224 \mathrm{e}-01$ & $1.0025 \mathrm{e}-13$ & 4 & 15 & 6.19 \\
\hline 8 & M4 0P I_n1 & 2028 & 3 & 3 & $2.61940 \mathrm{e}-01$ & $8.8036 \mathrm{e}-13$ & 4 & 14 & 8.71 \\
\hline 9 & M80PI_n1 & 4028 & 3 & 3 & $2.62898 \mathrm{e}-01$ & $3.0728 \mathrm{e}-13$ & 4 & 15 & 16.69 \\
\hline 10 & S10PI_n & 682 & 1 & 1 & $2.51602 \mathrm{e}-01$ & 0 & 4 & 14 & 3.81 \\
\hline 11 & S20PI_n & 1182 & 1 & 1 & $2.90430 \mathrm{e}-01$ & $9.2875 \mathrm{e}-12$ & 4 & 15 & 5.54 \\
\hline 12 & S40PI_n & 2182 & 1 & 1 & $2.98747 \mathrm{e}-01$ & $1.2982 \mathrm{e}-10$ & 4 & 18 & 10.26 \\
\hline 13 & S80PI_n & 4182 & 1 & 1 & $2.96722 \mathrm{e}-01$ & $3.9808 \mathrm{e}-13$ & 4 & 14 & 16.70 \\
\hline 14 & M10PI_n & 682 & 3 & 3 & $2.46511 \mathrm{e}-01$ & 0 & 5 & 18 & 5.82 \\
\hline 15 & M20PI_n & 1182 & 3 & 3 & $2.58224 \mathrm{e}-01$ & $4.0637 \mathrm{e}-13$ & 4 & 16 & 7.22 \\
\hline 16 & M4 0PI_n & 2182 & 3 & 3 & $2.61940 \mathrm{e}-01$ & $2.2161 \mathrm{e}-13$ & 4 & 15 & 10.05 \\
\hline 17 & M80PI_n & 4182 & 3 & 3 & $2.62898 \mathrm{e}-01$ & 0 & 4 & 15 & 16.92 \\
\hline 18 & bips98_606 & 7135 & 4 & 4 & $4.95158 \mathrm{e}-03$ & $2.3928 \mathrm{e}-12$ & 4 & 16 & 45.86 \\
\hline 19 & bips98_1142 & 9735 & 4 & 4 & $6.62334 \mathrm{e}-03$ & $5.5157 \mathrm{e}-13$ & 5 & 34 & 105.98 \\
\hline 20 & bips98_1450 & 11305 & 4 & 4 & $5.06614 \mathrm{e}-03$ & $5.0261 \mathrm{e}-13$ & 5 & 26 & 98.96 \\
\hline 21 & bips07_1693 & 13275 & 4 & 4 & $4.89792 \mathrm{e}-03$ & $1.1406 \mathrm{e}-13$ & 8 & 49 & 185.36 \\
\hline 22 & bips07_1998 & 15066 & 4 & 4 & $5.07449 \mathrm{e}-03$ & $1.1421 \mathrm{e}-13$ & 4 & 28 & 138.02 \\
\hline 23 & bips07_2476 & 16861 & 4 & 4 & $5.27485 \mathrm{e}-03$ & $1.2511 \mathrm{e}-11$ & 4 & 57 & 282.77 \\
\hline 24 & bips07_3078 & 21128 & 4 & 4 & $4.77453 \mathrm{e}-03$ & $7.1606 \mathrm{e}-14$ & 5 & 19 & 134.46 \\
\hline 25 & xingo_afonso_itaipu & 13250 & 1 & 1 & $2.46546 \mathrm{e}-01$ & $-3.0780 \mathrm{e}-14$ & 3 & 9 & 47.82 \\
\hline 26 & mimo8x8_system & 13309 & 8 & 8 & $1.87164 \mathrm{e}+01$ & $2.3477 \mathrm{e}-13$ & 4 & 24 & 109.42 \\
\hline 27 & mimo28x28_system & 13251 & 28 & 28 & $8.43042 \mathrm{e}+00$ & $-2.1586 e-14$ & 3 & 11 & 106.23 \\
\hline 28 & mimo46x46_system & 13250 & 46 & 46 & $4.86309 \mathrm{e}-03$ & $9.3221 \mathrm{e}-14$ & 3 & 11 & 166.15 \\
\hline
\end{tabular}

[3] —-, Real and Complex Stability Radii: A Survey, ser. Progress in Systems and Control Theory. Boston: Birkhäuser, 1990, vol. 6, pp. 119-162.

[4] L. Dai, Singular Control Systems, ser. Lecture Notes in Control and Information Sciences, M. Thoma and A. Wyner, Eds. Heidelberg: Springer-Verlag, 1989, vol. 118.

[5] P. Kunkel and V. Mehrmann, Differential-Algebraic Equations - Analysis and Numerical Solution, ser. Textbooks in Mathematics. European Mathematical Society, 2006.

[6] K. Zhou and J. D. Doyle, Essentials of Robust Control, 1st ed. Prentice Hall, 1998.

[7] P. Benner and M. Voigt, "A structured pseudospectral method for $\mathcal{H}_{\infty}$-norm computation of large-scale descriptor systems," Max Planck Institute Magdeburg, Tech. Rep. MPIMD/12-10, May 2012. [Online]. Available: http://www.mpi-magdeburg.mpg.de/preprints/

[8] R. Byers, "A bisection method for measuring the distance of a stable matrix to the unstable matrices," SIAM J. Sci. Stat. Comput., vol. 9, pp. 875-881, Sept. 1988.

[9] S. Boyd, V. Balakrishnan, and P. Kabamba, "A bisection method for computing the $H_{\infty}$ norm of a transfer matrix and related problems," Mathematics of Control, Signals, and Systems, vol. 2, no. 3, pp. 207219, Sept. 1989.

[10] N. A. Bruinsma and M. Steinbuch, "A fast algorithm to compute the $H_{\infty}$-norm of a transfer function matrix," Syst. Control Lett., vol. 14, no. 4, pp. 287-293, 1990.

[11] S. Boyd and V. Balakrishnan, "A regularity result for the singular values of a transfer matrix and a quadratically convergent algorithm for computing its $L_{\infty}$-norm," Syst. Control Lett., vol. 15, no. 1, pp. $1-7,1990$.

[12] P. Benner, V. Sima, and M. Voigt, " $\mathcal{L}_{\infty}$-norm computation for continuous-time descriptor systems using structured matrix pencils," IEEE Trans. Automat. Control, vol. 57, no. 1, pp. 233-238, Jan. 2012.

[13] _ "Robust and efficient algorithms for $\mathcal{L}_{\infty}$-norm computation for descriptor systems," in Preprints of the 7th IFAC Symposium on Robust Control Design, Jun. 2012, pp. 189-194.

[14] P. Benner, R. Byers, V. Mehrmann, and H. Xu, "Numerical computation of deflating subspaces of skew-Hamiltonian/Hamiltonian pencils," SIAM J. Matrix Anal. Appl., vol. 24, no. 1, pp. 165-190.

[15] V. Mehrmann, C. Schröder, and V. Simoncini, "An implicitly-restarted
Krylov subspace method for real symmetric/skew-symmetric eigenproblems," Lin. Alg. Appl., vol. 436, pp. 4070-4087, May 2012.

[16] N. Guglielmi, M. Gürbüzbalaban, and M. L. Overton, "Fast approximation of the $H_{\infty}$ norm via optimization of spectral value sets," May 2012, submitted.

[17] N. Guglielmi and M. L. Overton, "Fast algorithms for the approximation of the pseudospectral abscissa and pseudospectral radius of a matrix," SIAM J. Matrix Anal. Appl., vol. 32, no. 4, pp. 1166-1192, 2011.

[18] G. W. Stewart and J.-G. Sun, Matrix Perturbation Theory, ser. Computer science and scientific computing. Academic Press, 1990.

[19] J. Rommes, "Arnoldi and Jacobi-Davidson methods for generalized eigenvalue problems $A x=\lambda B x$ with singular B," Math. Comp., vol. 77, pp. 995-1015, 2008.

[20] J. Rommes and N. Martins, "Efficient computation of transfer function dominant poles using subspace acceleration," IEEE Trans. Power Syst., vol. 21, no. 3, pp. 1218-1226, Aug. 2006.

[21] — "Efficient computation of multivariate transfer function dominant poles using subspace acceleration," IEEE Trans. Power Syst., vol. 21, no. 4, pp. 1471-1483, Nov. 2006

[22] J. Rommes and G. L. G. Sleijpen, "Convergence of the dominant pole algorithm and Rayleigh quotient iteration," SIAM J. Matrix Anal. Appl., vol. 30, no. 1, pp. 346-363, Feb. 2008.

[23] P. Benner and M. Voigt, " $\mathcal{H}_{\infty}$-norm computation for large and sparse descriptor systems," May 2012, submitted.

[24] N. Martins, P. C. Pellanda, and J. Rommes, "Computation of transfer function dominant zeros with applications to oscillation damping control of large power systems," IEEE Trans. Power Syst., vol. 22, no. 4, pp. 1657-1664, Nov. 2007.

[25] F. Freitas, J. Rommes, and N. Martins, "Gramian-based reduction method applied to large sparse power system descriptor models," IEEE Trans. Power Syst., vol. 23, no. 3, pp. 1258-1270, Aug. 2008.

[26] Y. Chahlaoui and P. Van Dooren, "A collection of benchmark examples for model reduction of linear time invariant dynamical systems," NICONET e.V., Tech. Rep. SLICOT Working Note 2002-2, Feb. 2002. [Online]. Available: http://www.slicot.org/index.php?site= benchmodred 